\title{
INFLUÊNCIA DA DENSIDADE E DO TIPO DE RESINA NAS PROPRIEDADES TECNOLÓGICAS DE PAINÉIS OSB DE Pinus taeda L.
}

\author{
Leopoldo Karman Saldanha ${ }^{1}$, Setsuo Iwakiri ${ }^{2}$ \\ ${ }^{1}$ Eng. Florestal, M.Sc., Ge Quimica/ Brasceras, Barueri, SP, Brasil - 1karman123@yahoo.com.br \\ ${ }^{2}$ Eng. Florestal, Dr., Depto. de Engenharia e Tecnologia Florestal, UFPR, Curitiba, PR, Brasil - setsuo@ufpr.br
}

Recebido para publicação: 05/05/2008 - Aceito para publicação: 29/09/2008

\begin{abstract}
Resumo
Este trabalho foi desenvolvido com objetivo de avaliar a influência de dois níveis de densidade de chapa e três tipos de resinas nas propriedades de painéis OSB de Pinus taeda L. Os painéis foram produzidos com densidade nominal de 0,65 e $0,90 \mathrm{~g} / \mathrm{cm}^{3}$, utilizando resinas fenol-formaldeído (FF), melamina-ureia-formaldeído (MUF) e fenol-melamina-ureia-formaldeído (PMUF), em quantidade de $6 \%$ de sólido resinoso. O aumento na densidade dos painéis melhorou as propriedades de MOE e MOR paralelo, ligação interna e absorção de água, entretanto o inchamento em espessura 24 horas foi prejudicado. Não foram constatados os efeitos da densidade e dos tipos de resinas no MOE e MOR perpendicular. Os painéis produzidos com resina FF apresentaram melhores resultados para todas as propriedades avaliadas, com exceção de MOE e MOR perpendicular. Os resultados de MOE e MOR, obtidos para os painéis OSB produzidos com densidade nominal de 0,65 e $0,90 \mathrm{~g} / \mathrm{cm}^{3}$ e resinas FF, MUF e PMUF atendem ao requisito mínimo da norma CSA 0437 (1993).

Palavras-chave: Painéis OSB; resina fenol-formaldeído; fenol-melamina-ureia-formaldeído.
\end{abstract}

\begin{abstract}
Effects of density and kind of resin in the properties of OSB of Pinus taeda L. This research was developed with the objective to evaluate the effects of two levels of board density and three kinds of resin in the properties of OSB made from Pinus taeda $\mathrm{L}$. The boards were manufactured with nominal density of 0,65 and $0,90 \mathrm{~g} / \mathrm{cm}^{3}$, using phenol-formaldehyde (FF), melamina-urea-formaldehyde (MUF) and phenol-melamina-urea-formaldehyde (PMUF) resins, in amounts of $6 \%$ of solid contents. The increase in board density results in better properties of MOE and MOR parallel, internal bond and water absorption, however, the thickness sweeling 24 hours was wronged. Not observed the effects of density and kind of resin in the MOE and MOR perpendicular. The board produced with FF resin showed better results on all of the properties with exception of MOE and MOR perpendicular. The results of MOE and MOR obtained for OSB produced with nominal density of 0,65 and 0,90 $\mathrm{g} / \mathrm{cm}^{3}$ and FF, MUF and PMUF resins attends to minimum values required from CSA 0437 (1993). Keywords: OSB; phenol-formaldehyde; phenol-melamina-urea-formaldehyde resin.
\end{abstract}

\section{INTRODUÇÃO}

A produção de painéis de partículas orientadas (OSB) teve início na década de 70, nos EUA e Canadá, como produto de $2^{\mathrm{a}}$ geração de painéis estruturais waferboard. No Brasil, a primeira unidade industrial, localizada no município de Ponta Grossa, estado do Paraná, iniciou sua produção em 2002, com capacidade instalada de $350.000 \mathrm{~m}^{3} /$ ano.

As características tecnológicas dos painéis OSB diferem dos painéis aglomerados no que se refere aos seguintes fatores: (i) as dimensões e o formato das partículas são maiores e retangulares; (ii) as resinas utilizadas são a fenol-formaldeído e MDI (difenil-metano di-isocianato), com alta resistência à umidade; (iii) as partículas são orientadas numa mesma direção; (iv) o painel é formado em três camadas cruzadas, sendo as faces no sentido longitudinal e o miolo no sentido transversal (MARRA, 1992; MALONEY, 1993). De acordo com Maloney (1993), a constituição em camadas cruzadas confere aos painéis OSB maior estabilidade dimensional e melhor distribuição da resistência mecânica no sentido paralelo e perpendicular. Estudos realizados por Gouveia et al. (2000), com painéis de partículas do tipo strand, orientadas e não orientadas, demonstraram que os painéis de partículas orientadas apresentam módulo de elasticidade e módulo de ruptura superior em relação aos painéis de partículas não orientadas. 
Com relação à proporção entre as camadas externas e camada interna na composição do painel OSB, Iwakiri et al. (2003), concluíram na sua pesquisa que a proporção de 20:60:20 apresentou melhor balanço das propriedades de flexão estática nos sentidos paralelo e perpendicular, quando comparados aos painéis com proporção de 30:40:30.

A densidade dos painéis é um dos parâmetros mais importantes na produção de painéis reconstituídos de madeira. De acordo com Cloutier (1998), as indústrias canadenses produzem painéis OSB com densidade entre 0,63 e $0,67 \mathrm{~g} / \mathrm{cm}^{3}$. A influência da densidade dos painéis sobre as propriedades físicas e mecânicas é altamente significativa. Maloney (1993) e Moslemi (1974) afirmam que painéis com maior densidade apresentam maior resistência mecânica, entretanto a sua estabilidade dimensional é prejudicada. Os autores atribuem à maior quantidade de partículas de madeira e, consequentemente, à maior densificação e compressão do material durante o processo de formação do painel na prensa quente a causa principal para o aumento nas propriedades mecânicas e no inchamento em espessura dos painéis. Kelly (1977) explica que o inchamento em espessura é maior para painéis de maior densidade, devido à maior taxa de liberação de tensões de compressão impostas durante a prensagem. Zhang et al. (1998) encontraram um aumento nas propriedades de módulo de elasticidade, módulo de ruptura e ligação interna, com o aumento na densidade dos painéis de 0,60 a $0,70 \mathrm{~g} / \mathrm{cm}^{3}$. Wu (1999) constatou a mesma tendência de aumento nessas propriedades, com o aumento na densidade dos painéis na faixa de 0,55 a $1,15 \mathrm{~g} / \mathrm{cm}^{3}$.

A escolha de um determinado tipo de resina na produção de painéis de partículas de madeira está condicionada às condições de uso do produto e aos custos. As resinas fenol-formaldeído (FF) e difenilmetano di-isocianato (MDI) são as mais empregadas para produção de painéis OSB em função da necessidade de alta resistência à umidade (CLOUTIER, 1999). A colagem com a resina MDI resulta em adesão muito mais forte em relação a outros tipos de resina, entretanto a sua desvantagem é com relação à alta aderência com alumínio e outros tipos de aço, causando problemas com o sistema de prensagem (MARRA, 1992). Recentemente, começaram a surgir como alternativas as resinas compostas do tipo melamina-ureia-formaldeído (MUF) e fenol-melamina-ureia-formaldeído (PMUF), com a finalidade de melhorar a estabilidade dimensional dos painéis com menor custo de produção.

Este trabalho foi conduzido com o objetivo de avaliar a influência da densidade e do tipo de resina nas propriedades físicas e mecânicas de painéis de partículas orientadas OSB produzidos com madeira de Pinus taeda L.

\section{MATERIAL E MÉTODOS}

Foi utilizada, nesta pesquisa, madeira de Pinus taeda L. com 30 anos de idade, coletada aleatoriamente na serraria da Estação Experimental da UFPR na forma de tábuas, com dimensões de $180 \times 85 \times 25 \mathrm{~mm}$. Para a colagem foram utilizados três tipos de resinas: fenol-formaldeído (FF), melamina-ureia-formaldeído (MUF) e fenol-melamina-ureia-formaldeído (PMUF), doadas pela empresa Hexion Química S.A.

As partículas do tipo strand foram geradas num picador de disco rotativo com as seguintes dimensões nominais: comprimento de $85 \mathrm{~mm}$, largura de $25 \mathrm{~mm}$ e espessura de $0,7 \mathrm{~mm}$. As partículas foram secas ao teor de umidade médio de $3 \%$ e classificadas em peneira para a retirada de "finos". As resinas FF, MUF e PMUF, em percentagem de sólidos de 6\%, foram aplicadas sobre as partículas numa encoladeira rotativa de laboratório. $\mathrm{O}$ colchão de partículas foi formado em aparato orientador de partículas com a composição em camadas cruzadas face:miolo:face de 25:50:25. Após a pré-prensagem para melhor acomodação das partículas, os painéis foram prensados à temperatura de $180^{\circ} \mathrm{C}$, pressão específica de $40 \mathrm{kgf} / \mathrm{cm}^{2}$ e tempo de prensagem de 8 minutos. Os painéis foram produzidos com densidade nominal de $0,65 \mathrm{~g} / \mathrm{cm}^{3} \mathrm{e} 0,90 \mathrm{~g} / \mathrm{cm}^{3}$ e dimensões de 480 x 480 x $15 \mathrm{~mm}$. Foram produzidos três painéis por tratamento. As variáveis de estudo foram densidades dos painéis e tipos de resinas, conforme plano experimental apresentado na tabela 1.

Após a prensagem, os painéis foram acondicionados na câmara de climatização à temperatura de $20 \pm 2{ }^{\circ} \mathrm{C}$ e umidade relativa de $65 \pm 3 \%$, até a estabilização, e, posteriormente, foram confeccionados os corpos-de-prova para realização dos seguintes ensaios físico-mecânicos: absorção de água e inchamento em espessura após 2 e 24 horas de imersão em água ( 2 corpos-de-prova/painel), ligação interna ( 5 corposde-prova/painel) flexão estática (2 corpos-de-prova paralelo/painel e 2 corpos de prova perpendicular/painel). Os ensaios foram conduzidos de acordo com os procedimentos descritos na norma ASTM D 1037 (1996). Os resultados obtidos foram avaliados através de análise de variância e teste de Tukey ao nível de probabilidade de $95 \%$. 
Tabela 1. Plano experimental.

Table 1. Experimental design.

\begin{tabular}{lcc}
\hline Tratamento & Densidade do painel $\left(\mathbf{g} / \mathbf{c m}^{3}\right)$ & Tipo de resina \\
\hline T1 & 0,65 & FF \\
T2 & 0,65 & MUF \\
T3 & 0,65 & PMUF \\
T4 & 0,90 & FF \\
\hline
\end{tabular}

\section{RESULTADOS E DISCUSSÃO}

\section{Influência da densidade nas propriedades dos painéis OSB}

Na tabela 2 estão apresentados os resultados de módulo de elasticidade (MOE) e módulo de ruptura (MOR) no sentido paralelo e perpendicular dos painéis OSB produzidos com diferentes densidades e colagem com resina fenol-formaldeído.

Tabela 2. Influência da densidade do painel sobre os valores médios de MOE e MOR paralelo e perpendicular.

Table 2. Effect of board density on the average values of MOE and MOR parallel and perpendicular.

\begin{tabular}{|c|c|c|c|c|}
\hline \multirow{3}{*}{$\begin{array}{l}\text { Densidade do painel } \\
\left(\mathrm{g} / \mathrm{cm}^{3}\right)\end{array}$} & \multicolumn{4}{|c|}{ Módulo de elasticidade (MOE) - kgf/cm² } \\
\hline & \multicolumn{2}{|c|}{ Paralelo } & \multicolumn{2}{|c|}{ Perpendicular } \\
\hline & Média & CV (\%) & Média & CV $(\%)$ \\
\hline $0,65(\mathrm{~T} 1)$ & $60.699 \mathrm{~A}$ & 6,22 & $29.773 \mathrm{~A}$ & 28,28 \\
\hline \multirow[t]{2}{*}{$0,90(\mathrm{~T} 4)$} & $71.798 \mathrm{~B}$ & 12,31 & $30.874 \mathrm{~A}$ & 17,05 \\
\hline & \multicolumn{4}{|c|}{ Módulo de ruptura (MOR) - kgf/cm² } \\
\hline $0,65(\mathrm{~T} 1)$ & $445,1 \mathrm{~A}$ & 18,62 & $250,7 \mathrm{~A}$ & 38,02 \\
\hline $0,90(\mathrm{~T} 4)$ & $550,0 \mathrm{~B}$ & 11,29 & $315,8 \mathrm{~A}$ & 23,40 \\
\hline
\end{tabular}

Médias seguidas de mesma letra são estatisticamente iguais ao nível de probabilidade de $95 \%$.

$\mathrm{O}$ aumento na densidade do painel resultou em médias estatisticamente superiores de MOE e MOR no sentido paralelo. Por outro lado, no sentido perpendicular, a densidade do painel não influenciou de forma significativa nos valores médios de MOE e MOR.

A relação direta entre a densidade do painel OSB com o MOE e MOR em flexão estática no sentido paralelo é amplamente comentada na literatura, como influência da maior compactação do colchão de partículas durante o processo de prensagem dos painéis (MALONEY, 1993; MOSLEMI, 1974). Mendes (2001), para painéis OSB produzidos com densidades nominais de 0,65 e $0,80 \mathrm{~g} / \mathrm{cm}^{3}$, encontrou aumentos nos valores médios do MOE de 45.575 para $52.262 \mathrm{kgf} / \mathrm{cm}^{2}$ e do MOR de 411 para $476 \mathrm{kgf} / \mathrm{cm}^{2}$, respectivamente. Os valores médios de MOE e MOR obtidos nesta pesquisa estão acima do mínimo estabelecido pela norma CSA 0437 (1993), de 45.000 e $13.000 \mathrm{kgf} / \mathrm{cm}^{2}$ para o MOE e de 234 e $96 \mathrm{kgf} / \mathrm{cm}^{2}$ para o MOR, respectivamente para os sentidos paralelo e perpendicular.

Na tabela 3 estão apresentados os resultados de ligação interna dos painéis OSB produzidos com diferentes densidades.

Tabela 3. Influência da densidade do painel sobre os valores médios de ligação interna.

Table 3. Effect of board density on the average values of internal bond.

\begin{tabular}{llc}
\hline \multirow{2}{*}{ Densidade do painel $\left(\mathbf{g} / \mathbf{c m}^{\mathbf{3}}\right)$} & \multicolumn{2}{c}{ Ligação interna $\left(\mathbf{k g f} / \mathbf{c m}^{\mathbf{2}}\right)$} \\
\cline { 2 - 3 } & Média & $\mathbf{C V ~}(\boldsymbol{\%})$ \\
\hline $0,65(\mathrm{~T} 1)$ & $4,03 \mathrm{~A}$ & 29,73 \\
$0,90(\mathrm{~T} 4)$ & $5,80 \mathrm{~B}$ & 24,88 \\
\hline
\end{tabular}

Médias seguidas de mesma letra são estatisticamente iguais ao nível de probabilidade de $95 \%$.

O valor médio de ligação interna obtido para os painéis produzidos com densidade nominal de $0,90 \mathrm{~g} / \mathrm{cm}^{3}$ foi estatisticamente superior em comparação aos painéis produzidos com densidade nominal de $0,65 \mathrm{~g} / \mathrm{cm}^{3}$. Maloney (1993) afirma que a maior razão de compactação das partículas dos painéis com maior densidade influencia de forma positiva nos resultados de ligação interna. As médias obtidas para as 
duas diferentes densidades de painéis atendem ao requisito mínimo da norma CSA 0437 (1993), de 3,45 $\mathrm{kgf} / \mathrm{cm}^{2}$. Marin (2001) obteve também aumento significativo nos valores de ligação interna com aumento na densidade dos painéis de 0,65 para $0,80 \mathrm{~g} / \mathrm{cm}^{3}$.

$\mathrm{Na}$ tabela 4 estão apresentados os resultados de absorção de água e inchamento em espessura após 2 e 24 horas de imersão em água dos painéis OSB produzidos com diferentes densidades.

Tabela 4. Influência da densidade do painel sobre os valores médios de absorção de água e inchamento em espessura.

Table 4. Effect of board density on the average values of water absorption and thickness sweeling.

\begin{tabular}{lcccc}
\hline \multirow{2}{*}{ Densidade do painel $\left(\mathbf{g} / \mathbf{c m}^{3}\right)$} & \multicolumn{4}{c}{ Absorção de água (\%) } \\
\cline { 2 - 5 } & \multicolumn{3}{c}{$\mathbf{2}$ horas } & \multicolumn{2}{c}{$\mathbf{2 4}$ horas } \\
\cline { 2 - 5 } & Média & CV (\%) & Média & CV (\%) \\
\hline 0,65 (T1) & $50,58 \mathrm{~B}$ & 25,22 & $74,17 \mathrm{~B}$ & 11,95 \\
0,90 (T4) & $30,38 \mathrm{~A}$ & 32,17 & $58,38 \mathrm{~A}$ & 22,18 \\
\hline & \multicolumn{5}{c}{ Inchamento em espessura (\%) } \\
\hline 0,65 (T1) & $27,95 \mathrm{~A}$ & 13,98 & $34,65 \mathrm{~A}$ & 13,03 \\
0,90 (T4) & $29,43 \mathrm{~A}$ & 14,67 & $42,78 \mathrm{~B}$ & 9,47 \\
\hline
\end{tabular}

Médias seguidas de mesma letra são estatisticamente iguais ao nível de probabilidade de $95 \%$.

O aumento na densidade nominal dos painéis resultou em média estatisticamente inferior para absorção de água e superior para inchamento em espessura após 24 horas de imersão em água. Para 2 horas de imersão, as diferenças foram significativas apenas para absorção de água. Segundo Maloney (1993) e Moslemi (1974), a redução na absorção de água com aumento na densidade dos painéis está relacionada com a maior compactação da estrutura do painel, dificultando a absorção e penetração da água. Com relação ao inchamento em espessura, os autores atribuem como causa principal a liberação de maiores tensões de compressão impostas aos painéis com maior densidade durante o processo de prensagem a quente. A média de inchamento em espessura de 34,65\% obtida para os painéis com densidade de $0,65 \mathrm{~g} / \mathrm{cm}^{3}$ está compatível com os valores médios de 33,70 e $31,08 \%$ obtidos por Iwakiri et al. (2003) para painéis OSB de Pinus spp com densidade nominal de $0,70 \mathrm{~g} / \mathrm{cm}^{3}$.

\section{Influência do tipo de resina nas propriedades dos painéis OSB}

Na tabela 5 estão apresentados os resultados de módulo de elasticidade (MOE) e módulo de ruptura (MOR), no sentido paralelo e perpendicular, dos painéis OSB produzidos com diferentes tipos de resinas.

Tabela 5. Influência do tipo de resina sobre os valores médios de MOE e MOR - paralelo e perpendicular.

Table 5. Effect of kind of resin on the average values of MOE and MOR - parallel and perpendicular.

\begin{tabular}{|c|c|c|c|c|}
\hline \multirow{3}{*}{ Tipo de resina } & \multicolumn{4}{|c|}{ Módulo de elasticidade (MOE) - kgf/cm² } \\
\hline & \multicolumn{2}{|c|}{ Paralelo } & \multicolumn{2}{|c|}{ Perpendicular } \\
\hline & Média & CV (\%) & Média & CV (\%) \\
\hline FF (T1) & $60.699 \mathrm{~B}$ & 6,22 & $29.773 \mathrm{~A}$ & 28,28 \\
\hline MUF (T2) & $59.562 \mathrm{~B}$ & 9,70 & $28.171 \mathrm{~A}$ & 31,10 \\
\hline \multirow[t]{2}{*}{ PMUF (T3) } & $52.290 \mathrm{~A}$ & 16,06 & $27.743 \mathrm{~A}$ & 8,66 \\
\hline & \multicolumn{4}{|c|}{ Módulo de ruptura (MOR) - kgf/cm² } \\
\hline $\mathrm{FF}(\mathrm{T} 1)$ & $445,1 \mathrm{~B}$ & 18,62 & $250,7 \mathrm{~A}$ & 38,02 \\
\hline MUF (T2) & $310,6 \mathrm{~A}$ & 10,91 & $222,6 \mathrm{~A}$ & 41,75 \\
\hline PMUF (T3) & $291,4 \mathrm{~A}$ & 17,26 & $203,4 \mathrm{~A}$ & 16,24 \\
\hline
\end{tabular}

Médias seguidas de mesma letra são estatisticamente iguais ao nível de probabilidade de 95\%. FF: fenol-formaldeído; MUF: melamina-ureia-formaldeído; PMUF: fenol-melamina-ureia-formaldeído.

Os resultados de MOE no sentido paralelo indicam, para painéis produzidos com resina FF e PMUF, médias estatisticamente superiores em comparação aos painéis produzidos com resina MUF. Para o MOR no sentido paralelo, os painéis produzidos com resina FF apresentaram média estatisticamente superior em comparação aos painéis produzidos com resina PMUF e MUF. No sentido perpendicular, não 
foram constatadas diferenças estatisticamente significativas entre os painéis produzidos com diferentes tipos de resinas.

O desempenho inferior dos painéis produzidos com resina MUF nos ensaios de MOE e MOR no sentido paralelo foi verificado também na pesquisa realizada por Murakami (1999). Por outro lado, na comparação com os requisitos da norma CSA 0437 (1993), todos os resultados obtidos nesta pesquisa para o MOE e MOR, paralelo e perpendicular, estão acima dos valores mínimos de 45.000, 13.000, 234 e $96 \mathrm{kgf} / \mathrm{cm}^{2}$, respectivamente.

Na tabela 6 estão apresentados os resultados de ligação interna dos painéis OSB produzidos com diferentes tipos de resinas.

Os painéis produzidos com as resinas MUF e PMUF comportaram-se de forma semelhante, mas foram estatisticamente inferiores quando comparados com os painéis produzidos com resina FF. Na comparação com os requisitos da norma CSA 0437 (1993), somente os painéis com resina FF apresentaram valor médio superior ao mínimo de $3,45 \mathrm{kgf} / \mathrm{cm}^{2}$ estabelecido pela referida norma. Os resultados obtidos nesta pesquisa para as resinas MUF e PMUF foram inferiores aos obtidos por Mendes et al. (2007) para painéis OSB de clones de Eucalyptus spp., cujos valores foram de 3,58 e 3,92 kgf $/ \mathrm{cm}^{2}$, respectivamente para os dois tipos de resinas.

Tabela 6. Influência do tipo de resina sobre os valores médios de ligação interna.

Table 6. Effect of kind of resin on the average values of internal bond.

\begin{tabular}{llc}
\hline \multirow{2}{*}{ Tipo de resina } & \multicolumn{2}{c}{ Ligação interna $\left(\mathbf{k g f} / \mathbf{c m}^{2}\right)$} \\
\cline { 2 - 3 } & Média & $\mathbf{C V}(\boldsymbol{\%})$ \\
\hline FF (T1) & $4,03 \mathrm{~B}$ & 29,73 \\
MUF (T2) & $2,86 \mathrm{~A}$ & 31,41 \\
PMUF (T3) & $2,25 \mathrm{~A}$ & 35,65 \\
\hline Ḿ́di
\end{tabular}

Médias seguidas de mesma letra são estatisticamente iguais ao nível de probabilidade de $95 \%$.

FF: fenol-formaldeído; MUF: melamina-ureia-formaldeído; PMUF: fenol-melamina-ureia-formaldeído.

Na tabela 7 estão apresentados os resultados de absorção de água e inchamento em espessura após 2 e 24 horas de imersão em água dos painéis OSB produzidos com diferentes tipos de resinas.

Tabela 7. Influência do tipo de resina sobre os valores médios de absorção de água e inchamento em espessura.

Table 7. Effect of kind of resin on the average values of water absorption and thickness sweeling.

\begin{tabular}{|c|c|c|c|c|}
\hline \multirow{3}{*}{ Tipo de resina } & \multicolumn{4}{|c|}{ Absorção de água (\%) } \\
\hline & \multicolumn{2}{|c|}{2 horas } & \multicolumn{2}{|c|}{24 horas } \\
\hline & Média & CV (\%) & Média & CV (\%) \\
\hline FF (T1) & $50,58 \mathrm{~A}$ & 25,22 & $74,17 \mathrm{~A}$ & 11,95 \\
\hline MUF (T2) & $72,35 \mathrm{~B}$ & 11,05 & $82,39 \mathrm{~A}$ & 5,32 \\
\hline \multirow[t]{2}{*}{ PMUF (T3) } & $85,21 \mathrm{C}$ & 7,09 & $95,11 \mathrm{~B}$ & 6,15 \\
\hline & \multicolumn{4}{|c|}{ Inchamento em espessura (\%) } \\
\hline FF (T1) & $27,95 \mathrm{~A}$ & 13,98 & $34,65 \mathrm{~A}$ & 13,03 \\
\hline MUF (T2) & $31,30 \mathrm{~A}$ & 16,85 & $35,17 \mathrm{~A}$ & 19,98 \\
\hline PMUF (T3) & $48,10 \mathrm{~B}$ & 16,48 & $53,39 \mathrm{~B}$ & 16,50 \\
\hline
\end{tabular}

Médias seguidas de mesma letra são estatisticamente iguais ao nível de probabilidade de 95\%. FF: fenol-formaldeído; MUF: melamina-ureia-formaldeído; PMUF: fenol-melamina-ureia-formaldeído.

Os resultados de absorção de água e inchamento em espessura após 24 horas de imersão em água demonstram, para os painéis produzidos com resina FF e PMUF, médias estatisticamente iguais entre si e superiores em comparação aos painéis produzidos com resina MUF. Os resultados dos ensaios sugerem que a inclusão do componente fenol na fabricação de resina composta melhora as propriedades de absorção de água e inchamento em espessura de painéis OSB. Como referência, as médias obtidas para os painéis produzidos com resinas FF e PMUF estão dentro da faixa de valores de 31,08 e 33,70\% obtidos por Iwakiri et al. (2003) para painéis OSB de Pinus spp. produzidos com resina FF. 


\section{CONCLUSÕES}

Com base nos resultados obtidos nesta pesquisa, as seguintes conclusões podem ser apresentadas:

- O aumento na densidade dos painéis OSB melhorou as propriedades de MOE e MOR no sentido paralelo, ligação interna e absorção de água, entretanto houve um aumento no inchamento em espessura 24 horas.

- $\quad$ O MOE e MOR no sentido perpendicular não foram afetados pelo aumento na densidade dos painéis OSB.

- Os painéis produzidos com resina fenol-formaldeído (FF) apresentaram melhores resultados para todas as propriedades avaliadas, com exceção de MOE e MOR no sentido perpendicular.

- Entre as resinas alternativas, a fenol-melamina-ureia-formaldeído (PMUF) foi a que apresentou resultados satisfatórios para o MOE no sentido paralelo, absorção de água e inchamento em espessura 24 horas.

- $\quad$ Os resultados de MOE e MOR no sentido paralelo e perpendicular dos painéis OSB produzidos com densidade de 0,65 e $0,90 \mathrm{~g} / \mathrm{cm}^{3}$ e resinas FF, PMUF e MUF foram superiores ao requisito mínimo da norma CSA 0437 (1993).

- A pesquisa demonstrou a viabilidade de produção de painéis OSB de alta densificação visando aplicações específicas. A resina PMUF apresenta potencial para utilização na produção de painéis OSB.

\section{REFERÊNCIAS}

AMERICAN SOCIETY FOR TESTING AND MATERIAL. ASTM D 1037: Standard methods of evaluation the properties of wood-base fiber and particle panel materials. Philadelphia, 1996.

CANADIAN STANDARDS ASSOCIATION. CSA 0437.0 - 93: OSB and Waferboard.. Ontario: 1993.18 p.

CLOUTIER, A. Oriented strandboard (OSB): raw material, manufacturing process, properties of woodbase fiber and particle materials. In: INTERNATIONAL SEMINAR ON SOLID WOOD PRODUCTS OF HIGH TECHNOLOGY, 1, 1998, Belo Horizonte. Anais... Belo Horizonte: SIF, 1998. p. 173-185.

IWAKIRI, S.; MONTEFUSCO, A. R. G.; ZAMBLOSKY, K. M.; SIQUEIRA, K. P.; SALDANHA, L. K.; SOUZA, M. A. M. Produção de chapas de partículas "strand" com inclusão laminar - "com-ply". Floresta e Ambiente, Seropédica, v. 10, n. 2, p. 30-35, ago./dez. 2003.

KELLY, M. W. Critical literature review of relationships between processing parameters and physical properties of particleboard. 1977. 66 p. Madison: U.S. For. Prod. Lab. (General Technical Report FPL, 10).

MALONEY, T. M. Modern particleboard e dry-process fiberboard manufacturing. 2 ed. São Francisco: M. Freeman , 1993. 689 p.

MENDES, L. M Pinus spp na produção de painéis de partículas orientadas (OSB). 103 p. Tese (Doutorado em Ciências Florestais) - Setor de Ciências Agrárias, Universidade Federal do Paraná, Curitiba, 2001.

MURAKAMI, K. Manufacture and properties of three-layered particleboards with oriented face strands of veneers I. Journal of Wood Science, Tokyo, v. 45, n. 5, p. 395-402, 1999.

MENDES, S. A.; MENDES, L. M.; CHAVES, M. D.; MORI, F. A.; SILVA, J. R. M.; TRUGILHO, P. F. Utilização de resinas alternativas na produção de painéis OSB de clones de Eucalyptus spp. Cerne, Lavras, v. 13, n. 3, p. 257-263, jul./set. 2007.

MARRA, A. A. Technology of wood bonding: principles in practice. New York: Van Nostrand Reinhold, 453 p. 1992.

MOSLEMI, A. A. Particleboard vol. 1: Materials. London: Southern Illinois University Press, 1974, 244 p.

WU, Q. In-plane dimensional stability of oriented strand panel: effect of processing variables. Wood and Fiber Science. Madison, v. 31, n. 1, p. 28-40, 1999.

ZHANG, M.; WONG, E. D.; KAWAI, S.; KNON, J. H. Manufacture and properties of high-performance oriented strand board composite using thin strands. Journal of Wood Science, Tokyo, v. 44, n. 3, p. 191-197, 1998. 